\title{
Study on Detection Algorithm of Moving Housed-pig Targets Based on Back- ground Subtraction Method
}

\author{
Zhenye $\mathrm{Li}^{1}$ Longqing $\mathrm{Sun}^{2}$ Qiong $\mathrm{Wu}^{3}$ \\ ${ }^{1}$ Affiliation (China Agricultural University, Beijing 100083)
}

\begin{abstract}
In this article, a pig detection algorithm based on self-adaptive background subtraction is presented, by applying the improved background subtraction algorithm to target detection of moving objects in the static background. The method of averaging the values of Multi-Frame images is used to avoid error detection object in background testing due to the moving objects appearing in the first frame. There will be a quick response after the dynamic changes of the background by using Surendra background updating algorithm to update the background. Experiment results showed that the algorithm is available and efficient, and can meet the requirements of dynamic update of background.
\end{abstract}

Keywords: background, background subtraction method, object detection, background initiation, background updating

\section{Introduction}

Moving object detection is to detect the target that interests people in video image sequence. Commonly methods of moving object detection: Frame difference method (PARAGIOS N et al, 1996)(Ye Z et al, 2002), Background subtraction method(MCKENNA S et al, 2000) ( MONNET A et al, 2003)and Optical flow method.There are several ways of the moving target detection, so far, there is not a generic algorithm which can be applicable to all kinds of specific situations. Due to the objective conditions of the practical application occasions are different, the use of the moving target detection method is also different.

Background Subtraction Method (STAUFFER C et al,1999) is one of the most commonly used methods for moving target detection and it will divide the image into two parts foreground and background. Firstly, model the background, then use the difference of current frame and the background image to detect the moving region. This method can get more precise information of the moving target, but it is particularly sensitive to the changes in the scene. In practical applications, this method is always needed to commit to the performance of all kinds of background models and add the update mechanism for background image to reduce the impact of the dynamic change in the scene for the accurate segmentation. For example Haritaoglu et al. (2000) used the minimum, maximum intensity value and the maximum time difference to statistically model each pixel in the scene and update the background periodically. Karmann, Brandt et al. (1990) built the self-adaptive background model based on Kalman Filtering method to adapt to the time change of weather and light in the scene. 
This article combines the behavior characteristics of pigs with the characteristics of the video image, applying the improved background subtraction algorithm into housed pigs moving target detection in static background, and builds the detection algorithm of moving housed-pig targets based on self-adaptive background subtraction method. In order to reduce the distortion of the initial background, we use the method of calculating the average value of multi-frame image to avoid the detection errors due to the moving objects appearing in the first frame; by using Surendra background updating algorithm to update the background, the updated background can reflect the dynamic changes timely in the background.

\section{Experimental methods}

\subsection{Target detection method}

Because that background subtraction method has good real-time performance and scalability, in this article we choose the background subtraction method to achieve the detection of moving targets, and improve it on the basis of principles of the background subtraction method to adapt to application requirements of this system.

Suppose that use $\mathrm{B}_{\mathrm{k}}(\mathrm{x}, \mathrm{y})$ to represent the established background image. From the first frame to the $50^{\text {th }}$ frame, use the method of cumulative average to estimate for a complete background image $\mathrm{B}_{50}(\mathrm{x}, \mathrm{y})$.And each frame after the $51^{\text {st }}$ frame image, if the noise impact is not considered, can be expressed as follows:

$$
f_{k}(x, y)=M_{k}(x, y)+B_{k}(x, y)
$$

Expression (1) said that there are two parts in the $\mathrm{k}^{\text {th }}$ frame: Moving object region $M_{k}(x, y)$ and the background re- gion $\mathrm{B}_{\mathrm{k}}(\mathrm{x}, \mathrm{y})$. Obviously, the background of the $\mathrm{k}^{\text {th }}$ frame $\mathrm{B}_{\mathrm{k}}(\mathrm{x}, \mathrm{y})$ is one part of a complete background. We can divide the complete the background $\mathrm{B}_{50}(\mathrm{x}, \mathrm{y})$ into two parts of the background area in the $\mathrm{k}^{\text {th }}$ frame and the covering background by moving object in the $\mathrm{k}^{\text {th }}$ frame, namely,

$$
\mathrm{B}_{50}(\mathrm{x}, \mathrm{y})=\mathrm{B}_{\mathrm{k}}(\mathrm{x}, \mathrm{y})+\mathrm{C}_{\mathrm{k}}(\mathrm{x}, \mathrm{y})
$$

Where $C_{k}(x, y)$ represents the covering background by moving object in the $\mathrm{k}^{\text {th }}$ frame, $\mathrm{C}_{\mathrm{k}}(\mathrm{x}, \mathrm{y})$ and the moving object in the $\mathrm{k}^{\text {th }}$ frame are entirely superposition in the space position.

Make the $k^{\text {th }}$ frame image $f_{k}(x, y)$ and the complete background do the difference operation to get the difference image as folows:

$$
D_{k}(x, y)=f_{k}(x, y)-B_{50}(x, y)=M_{k}(x, y)-C_{k}(x, y)
$$

Expression (3) shows that the difference image of the $\mathrm{k}^{\text {th }}$ frame image $\mathrm{f}_{\mathrm{k}}(\mathrm{x}, \mathrm{y})$ and the complete background $\mathbf{B}_{50}(\mathrm{x}, \mathrm{y})$ consists of the moving object and the background covered by moving object. Because of the complete superposition of $M_{k}(x, y)$ and $C_{k}(x, y)$ in the space position, the difference image does not consist of the exposed (or covered) background region caused by the moving target.

The main workflow of the background subtraction method is shown in Fig. 1.

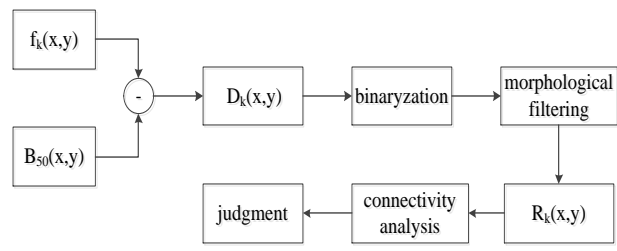

Fig. 1: The basic principle process of the background subtraction method 
Firstly, calculate the difference between the background image $\mathrm{B}_{50}(\mathrm{x}, \mathrm{y})$, and the current frame image $f_{k}(x, y)$ to obtain the difference image $\mathrm{D}_{\mathrm{k}}(\mathrm{x}, \mathrm{y})$ and then process the difference image with the method of Binary and morphological filtering to obtain the image $\mathrm{R}_{\mathrm{k}}(\mathrm{x}, \mathrm{y})$, and analyze the image $\mathrm{R}_{\mathrm{k}}(\mathrm{x}, \mathrm{y})$ using the area connectivity analysis. When the area of a connected region (number of pixels) is bigger than a given threshold (Zhang Xin, Li Daoliang et al, 2011), consider that we detect the moving targets, and consider that the connected region is the detected target image.

\subsection{Background modeling}

In general, the background subtraction method can effectively extract moving targets, and reflect the characteristic data integrally. But in the face of the scene changes, light factors and unrelated events interfere and other complicated situations, it shows sensitivity. Background image has a strong correlation with time and environment, with changes of time and environment, the changeable background image may cause a large wrong result of the moving target detection. For example, slow changes in light result in a background image to exceed motion detection range, and may make part of the background misclassify to the moving objects. So we should establish a suitable background model which could adapt to changes in external factors to solve the interference of the moving object detection brought by the above factors.

1. The establishment of initial background

General background subtraction method always selects the first frame of the video camera image as a background, and makes a difference between the follow of the frame and the background. And there is also a background updating in followup steps. If there is a moving target in the first frame, then it will be a part of the background, while the background in the original location will become the target to be detected in the next frames. In order to avoid the initial background distortion, we use multi-frame statistical average (Cutler R et al, 1998; Cucchiara R et al, 2003) method in this article. The establishment of the initial background takes the method of doing the average of the initial 50 frames. Namely, make a accumulating on a continuous 50 frame images to calculate the average:

$$
B_{0}=B_{50}(x, y)=\frac{1}{50}\left(f_{1}+f_{2}+\ldots+f_{50}\right)
$$

Where $\mathrm{f}_{1}, \mathrm{f}_{2}, \ldots, \mathrm{f}_{50}$ are the $1^{\text {st }}$ frame to the $50^{\text {th }}$ frame.

2. Background update

1) Background updating principle

The establishment and update of background model will affect the results of motion detection. At present, in order to reduce the impact made by the dynamic scene changes on the results of moving target detection, many researchers are committed to establishing different background models to make a dynamic update of the background model. And the following two principles (Liu Y et al, 2002) should be noted in the background model updates: the response speed of the background model to background changes should be fast enough, the background model should have a strong antiinterference ability to the moving objects. Fig. 2 shows a flowchart of the background model based on background subtraction method. 


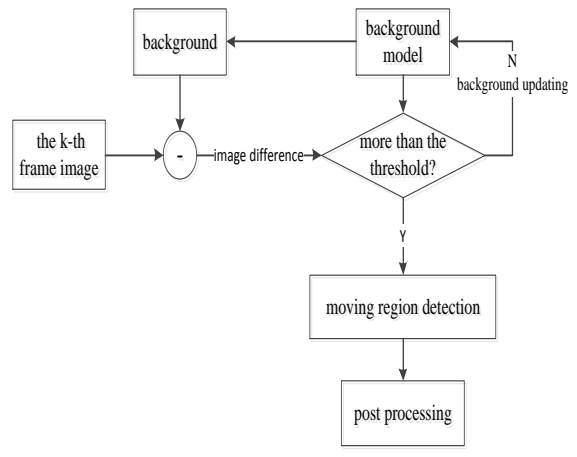

Fig. 2: background model work Schematic based on the background subtraction method

2) Background updating method

The advantages of the background subtraction method are conducive to realtime calculation, simple method, easy to engineering realization, and the drawback is that it has a high demand for the update of the background image ,and it is vulnerable to the environmental light interference which can affect the robustness.

Background update is requested to adapt to environmental changes as soon as possible, including changes in the external environment factors (such as light, weather changes, etc.) and background information itself changes (for example some targets move into the background and become the background, or targets originally belonging to the background come out to be a target). In this subject, we use the the Surendra background update algorithm to ensure real-time updates of the background. The algorithm can be divided into the following steps:

(1) Make the statistical average of the first 50 frame images as the initial background $\mathrm{B}_{0}$.

(2) Make the current frame and initial background do difference to get difference image, and do a binarization processing.

(3) use binary image $\mathrm{D}_{\mathrm{i}}(\mathrm{x}, \mathrm{y})$ to update the background image $\operatorname{TempB}_{\mathrm{i}}(\mathrm{x}, \mathrm{y})$, $\mathrm{f}_{\mathrm{i}}(\mathrm{x}, \mathrm{y})$ is the current frame gray image, namely

$\operatorname{TempB}_{i}(x, y)= \begin{cases}B_{i-1}(x, y), & D_{i}(x, y)=0 \\ f_{i}(x, y), & D_{i}(x, y)=1\end{cases}$

(4) makes the data TempB ${ }_{i}$ obtained by steps (3) do weighted stacking to the original background image, we can see from the above equation, TempB $\mathrm{i}(\mathrm{x}, \mathrm{y})$ is the current frame after removing the target pixel which can do weighted stacking to background model. The update algorithm flowchart is shown in Fig. 3.

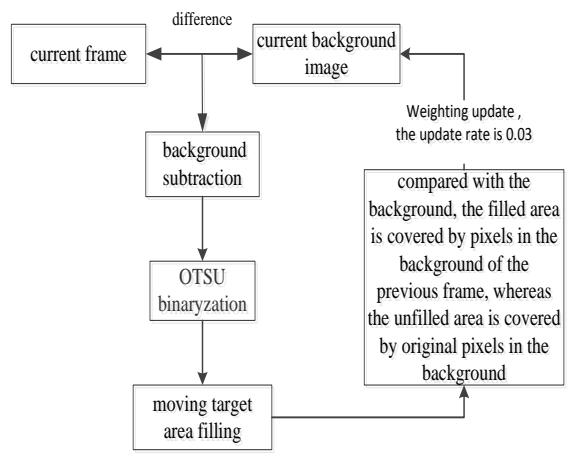

Fig. 3: the algorithm flowchart based on the Surendra background update

\section{Results and discussion}

(1) In this paper, we detect the moving target by the improved background subtraction algorithm. The experimental results show that real-time and scalability of the method can better meet the application requirements. To construct the initial frame, we use the initial 50 images to get pixel-average. If the first frame has a moving object, this method can avoid detecting the wrong object blocks.

(2) The Surendra background update algorithm is used to obtain the background image adaptively. This algorithm keeps the background of the moving object area as the same; meanwhile, it re- 
places the background of the non-moving object area by the current frame. Thus, after a period of time it can extract the background image.

\section{Conclusions}

In this paper, considering the characteristics of pigs' behavior and video images, we have established a detection algorithm of the movement of live pigs based on self-adaptive background subtraction method. In order to reduce the distortion of the initial background, we have adopted a multi-frame statistical average method to avoid the background detection error when the first frame has a moving object. We use Surendra background update algorithm to update the background. Background updated in a timely manner to reflect the dynamic changes in the background. The experiments show that the pig detection algorithm meets the demand of rapid extraction of the background image, small amount of calculation and dynamical update of background.

ACKNOWLEDGMENTS: This paper was supported by the National High Technology Research and Development Program (863 Plan) 2013AA102306, Shandong Province Self-innovation Projects (2012CX90204), and the National Science and Technology Supporting Plan Project (2012BAD35B06).

\section{References}

[1]PARAGIOS $\mathrm{N}$, and TZIRITAS $\mathrm{C}$, "Detection and Location of Moving Objects Using Deterministic Relaxation Algorithms,'Proceedings of the 13th International Conference on Pattern Recognition.1996, 1(1):201-205.

[2]Ye Zhongfu, and Li Houqiang, "a new method for moving target detection based on order cumulants," Computer Engineering and Applications. 2002,4:102-105.
[3] MCKENNA S, JABRI Z, and DURIC $\mathrm{Z}$, "Tracking groups of people," Computer Vision and Image Understanding.2000, 80(1):42-56.

[4]MONNET A, MITTAL A, and RAMESH V, "Background modeling and subtraction of dynamic scenes," Proceeding of Ninth IEEE International Conference on Computer Vision.2003, 2:13051312.

[5]Chen Yan, and Liu Kaihua, "Dynamic context of moving target detection and tracking communications technology," 2003,12:104.105.

[6] Zhang Xin, Li Daoliang, "Fast Segmentation of High-resolution Color Images of Cotton Foreign Fibers," Transactions of the Chinese Society for Agricultural Machinery, 2011.

[7] Cucchiara R, Piccardi.M, and Prati A, "Detecting moving objects, ghosts and shadows in video streams," IEEE Transaction on Pattern Analysis and Machine Intelligence.2003, 25(10):1337-1342.

[8] Haritaoglu I, Harwood D, Davis L, W4: "Real-time surveillance of people and their activities,"IEEE Transactions on Pattern Analysis and Machine Intelligence. (2000)22,809-830.

[9] Karmann K, and Brant A, "Moving object recognition using all adaptive background memory," In: Cappellini V, ed, Time-Varying Image Processing and Moving Object Recognition, Amsterdam: Elsevier Science, (1990), 289-296.

[10] Cutler R, and Davis L, "View-based detection,'In: Proceedings of International Conference on Pattern Recognition. Brisbane, Australia, 1998:495-500. 